intervals of $15 \mathrm{~min}$. The volume concludes with reproductions of the complete series of magnetograms from April 30, 1957, to December 31, 1958.

In all, this work presents the results of a prodigious amount of labour. It is well set-out, and the typography is excellent. The Royal Society and the authors are to be congratulated on their fine achievement.

${ }^{1}$ vature, 188, 529 (1960).

R. STONELEY

\section{THE TEACHING OF QUANTUM MECHANICS}

Introduction to Quantum Mechanics

By Prof. Robert H. Dicke and Dr. James P. Wittke. Pp. $x i+369$. (Reading, Mass., and London: AddisonWesley Publishing Company, Inc., 1960. 66s.)

Problems in Quantum Mechanics

By I. I. Gol'dman, V. D. Krivchenkov, V. I. Kogan and V. M. Galitskii. Pp. iii +394 . (London: Infosearch Limited, 1960. Distributed by Cleaver-Hume Press, Ltd.) $42 s$.

Problems in Quantum Mechanics

By I. I. Gol'dman and V. D. Krivchenkov Edited by Prof. B. T. Geilikman. Translated from the Russian by E. Marquit and E. Lepa. Authorized revised edition. Pp. viii +275 . (London and New York: Pergamon Press, 1961.) $50 s$.

ATER many years of famine in the supply of A quantum mechanics text-books, there is now an abundance and more titles keep appearing. It is possible therefore to be fairly eritical in deciding which text-books to recommend to students. Quantum theory is gradually taking a larger part in undergraduate training in general and theoretical physics, and the type of book required at the undergraduate level is not, for example, Dirac's, which is too difficult, or that of Landau and Lifshitz which is too big and thorough.

Prof. Dicke and Dr. Wittke have written their text for the first postgraduate physics year at Princeton, and it covers much the same ground as that at many British universities (it should be all), in the third year of honours physics. All the essentials of quantum theory are covered, and the late chapters of the book cover the effects of strong fields on atoms, scattering theory, the effect of statistics on the two and many-body systems, and on quantum statistical mechanics. There is a surprising and serious omission from the contents since the uncertsinty relation between energy and time is never mentioned. On the whole it is well written, with the difficultios that a beginner experiences well aired. Useful sets of examples are appended to each chapter. But it can be criticized in that several mathematical statements are not precise, and the same lack of precision shows up in several of the discussions of points of principle. For example: Why should Hermites polynomials have to terminate? Why should they be polynomials at all? Why should the reader accept, without proof, that $(\exp i k r) / r$ is the Green function for scattering and not $(\exp -i k r) / r$ ? The discussion of orbital and spin angular momentum make these appear only trivially different, and in discussion of what Dirac calls the sehrödinger and Heisenberg 'pictures', the word representation is confusingly used to mean picture as well as its mathematical meaning with respect to a basis. There are several other minor inaccuracies and obscurities, and a general tautening up of statements could be made throughout the book without making it longer or more difficult. In spite of these drawbacks it is an attractive book and beautifully produced.

The two collections of examples present a curious situation for they are effectively the same book, being translations of a set of problems with full solutions, used by Drs. Gol'dman and Krivchenkov in teaching a course of quantum mechanics based on Landau and Lifshitz's text. The volume from Infosearch has added further problems from a comparable set by Kogan and Galitskii, while that from Pergamon is based solely on Gol'dman and Krivehenkov, though from an extended and revised version. The latter states it is the 'authorized version', which may account for the price difference. As it stands the Infosearch edition is considerably larger and clearly the 'best buy'. These problems cover much more ground than say Dicke and Wittke, and are suitable for theoretical specialists. With the book of Landau and Lifshitz they throw up a powerful challenge. This is how quantum mechanics is taught to theorists in Moscow. Are British theorists taught as thoroughly ? Moreover, in those universities where they are, do the students also reach the same standard in hydrodynamics, physical kineties, elasticity, etc., as the introductions to the Landau and Lifshitz texts assure us the Russian theorists do. Regretfully one must give the answer that scarcely anywhere in Britain is this standard aimed at, let alone achieved.

$$
\text { S. F. EDwards }
$$

\section{THEORY AND TABLES OF THE BLUE SKY}

Tables related to Radiation emerging from a Planetary Atmosphere with Rayleigh Scattering By Kinsell L. Coulson, Jitendra V. Dave and Zdeněk Sekera. Pp. $\mathrm{x}+548$. (Berkeley and Los Angeles: University of California Press; London: Cambridge University Press, 1960.) 116s. net.

$\mathrm{O}$ $\mathrm{NE}$ of the best-known of the many fundamental contributions to science made by Lord Rayleigh during his long career was his discovery of the law of molecular scattering (named after him) and its application to the explanation of the colour and polariza. tion of the sunlit sky. The problem of the diffuse reflexion and transmission of sunlight in an atmosphere scattering it in accordance with Rayleigh's law has since become one of the classical problems of mathematical physics, of particular interest to meteorology. Many efforts were made toward its solution-culminating in the well-known work by Chandrasekhar, who succeeded in constructing its exact solution in terms of the so-ralled $X$ - and $Y$-functions satisfying four pairs of simultaneous integral equations-but, alas, these equations turned out to be non-linear and of the type which resolutely defies any possibility of an analytical solution.

Yet, in order to learn what these functions are capable of revealing, it is necessary to evaluate them numerically and insert in various relevant expressions. The construction (by successive approximations) of numerical solutions of the respective equations has indeed been undertaken recently by the authors of 